\title{
La partie radiologique de l'éthique nucléaire suisse
}

\author{
S. PRETRE* \\ (Manuscrit reçu le 15 mars 1983)
}

\section{RÉSUMÉ}

Les directives officielles de la Confédération suisse (directives R-11) pour la protection des personnes auprès des centrales nucléaires sont reproduites dans le texte en annexe relatif aux objectifs de la protection contre les rayonnements ionisants pour les personnes travaillant dans les centrales nucléaires et pour les personnes du public.

\section{ABSTRACT}

The official regulations of the Swiss Confederation (R-11) for the protection of persons near nuclear power plants are stated in a text about the aims of protection against ionising radiation for nuclear power plant workers and individuals of the public.

Les directives officielles de la Confédération suisse (directives R-11) pour la protection des personnes auprès des centrales nucléaires sont reproduites dans le texte en annexe relatif aux objectifs de la protection contre les rayonnements ionisants pour les personnes travaillant dans les centrales nucléaires et pour les personnes du public.

Les deux limites importantes qui ressortent de ce texte sont :

a) 400 hommes.rems par an et par réacteur pour l'ensemble du personnel (voir § 4.3) ;

b) $20 \mathrm{mrem} / \mathrm{an}$ aux personnes de la population par suites des rejets de substances radioactives (voir $\S 3.1$.a).

\begin{tabular}{|c|c|c|c|}
\hline & & \multicolumn{2}{|r|}{ Limites de rejet } \\
\hline & & $\begin{array}{c}\text { annuelle } \\
\mathrm{Ci} / \mathrm{an}\end{array}$ & $\begin{array}{l}\text { à court } \\
\text { terme }\end{array}$ \\
\hline \multirow{3}{*}{ 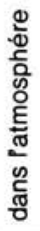 } & $\begin{array}{l}\text { gaz rares (en équivalents de } \\
\left.{ }^{133} \mathrm{Xe}\right)\end{array}$ & 30000 & $600 \mathrm{Ci} /$ jour \\
\hline & $\begin{array}{l}\text { Aérosols de période supérieure à } \\
8 \text { jours }\left(\beta, \gamma \text { sans }{ }^{13^{1}} \mathrm{l}\right)\end{array}$ & 0,25 & $0,02 \mathrm{Ci} /$ semaine \\
\hline & lode 131 & 0,2 & $0,05 \mathrm{Ci} /$ semaine \\
\hline \multirow{2}{*}{$\frac{\text { J }}{\Phi}$} & Tritium & 2000 & \multirow{2}{*}{$\begin{array}{l}5 \text { fois la concentration maximale } \\
\text { admissible pour les personnes } \\
\text { professionnellement exposées }\end{array}$} \\
\hline & $\begin{array}{l}\text { Reste des rejets liquides (calculés } \\
\text { pour un mélange } 4 \text { fois moins } \\
\text { radiotoxique que }{ }^{137} \mathrm{Cs} \text { ) }\end{array}$ & 5 & \\
\hline
\end{tabular}

* Division principale de la sécurité des installations nucléaires, Division de Radioprotection, $\mathrm{CH}-5303$ Würenlingen, Suisse. 
La dose b) ci-dessus conduit les autorités à décréter des limites de rejet qui sont officialisés dans l'autorisation d'exploitation. En guise d'exemple, nous reproduisons ici les limites de rejet actuellement en vigueur pour la centrale nucléaire de Gösgen-Däniken (Réacteur à eau sous pression de Kraftwerk-Union de $970 \mathrm{MWe}$ ).

Depuis la mise en vigueur des directives R-11, en 1978, l'expérience a montré que les objectifs prévus sont atteignables, et que les efforts nécessaires à leur réalisation ne sont pas exagérés. II faut, cependant, remarquer que la limite de 400 hommes.rems par an et par réacteur pour l'ensemble du personnel est basse et qu'elle exige une planification très serrée de tous les engagements en zone contrôlée.

\section{ANNEXE}

Commission fédérale pour la sécurité des installations atomiques (CSA), Commission fédérale de surveillance de la radioactivité (CSR), Division pour la sécurité des installations nucléaires (DSN).

Objectifs de la protection des personnes contre les radiations ionisantes dans la zone d'influence des centrales nucléaires.

Directives pour installations nucléaires R-11, juillet 1978, révision mai 1980).

\section{BUT DU PRESENT DOCUMENT}

Selon la loi fédérale du 23 décembre 1959 sur l'utilisation pacifique de l'énergie atomique et la protection contre les radiations, art. 7 et 10 , les organes de sécurité de la Confédération doivent établir si toutes les mesures que l'on peut raisonnablement exiger en vertu de l'état de la science et de la technique pour la protection des personnes, des biens d'autrui et des droits importants sont prises. Autant dans la procédure d'autorisation qu'au cours de l'exploitation, ces organes doivent donc examiner, en particulier, si le risque d'irradiation des personnes dans la zone d'influence des centrales nucléaires est maintenu à un niveau suffisamment bas.

Le présent document indique les bases sur lesquelles se fondent les organes de sécurité pour l'accomplissement de cette tâche.

\section{CADRE}

Pour limiter l'irradiation des personnes dans la zone d'influence des centrales nucléaires on applique d'abord les prescriptions de l'ordonnance du 30 juin 1976 concernant la protection contre les radiations (OPR). II est également tenu compte des recommandations de la CIPR(1), formulées en particulier dans ses publications nos 7,22 et 26 .

On peut dégager de ces documents les règles fondamentales suivantes:

a) Les limites de dose par individu de la population fixées dans l'OPR, art. 44, les limites dérivant de l'art. 107 ainsi que les doses maximales admissibles pour des personnes exposées aux radiations dans l'exercice de leur profession (art. 32 à 36) ne doivent pas être dépassées.

b) L'irradiation des personnes aux alentours d'une centrale nucléaire ne doit pas dépasser une certaine fraction de la valeur limite de la dose par individu de la population (quota pour centrales nucléaires).

(1) CIPR : Commission internationale de protection radiologique. 
c) Les doses collectives doivent être limitées aussi bien pour la population que pour le personnel d'une centrale nucléaire.

d) L'irradiation des personnes doit être maintenue à un niveau suffisamment bas pour qu'une réduction supplémentaire ne se justifie plus (as low as reasonably achievable " ALARA" (1).

\section{PROTECTION DE LA POPULATION}

\subsection{Limitation de l'irradiation durant l'exploitation normale et en cas de dérangement $\left({ }^{2}\right)$}

En tenant compte des règles fondamentales énoncées à l'alinéa 2 , on applique ce qui suit :

a) Dans la procédure d'autorisation, les taux d'émission maximaux de substances radioactives dans l'atmosphère et dans le cours d'eau récepteur doivent être limités de telle sorte que:

- les prescriptions de l'art. 107 OPR soient respectées;

- la dose annuelle par individu de la population ne dépasse pas 20 mrem (voir en 5.2. le calcul de la dose);

- les émissions prévues soient réduites plus strictement encore dans la mesure où le permet l'état de la technique.

Sur l'ensemble de l'année, les émissions prévues doivent être si faibles qu'une réduction plus draconienne ne se justifie plus.

b) Outre la limitation des émissions annuelles, la procédure d'autorisation prévoit, dans les conditions d'exploitation normales, une limitation pour des périodes plus courtes.

c) Les limites d'émissions fixées dans la procédure d'autorisation ne sont soumises à aucune restriction supplémentaire.

d) Le calcul des atteintes ("immissions") occasionnées par les émissions doit se fonder sur des modèles et hypothèses réalistes.

e) Le rayonnement direct provenant d'une centrale nucléaire ne doit causer en aucun lieu accessible, situé hors du terrain clôturé, un débit de dose ambiante supérieur à 10 mrem par semaine.

On procédera à une limitation supplémentaire de ce débit de dose ambiante, s'il faut prévoir que la dose accumulée annuelle aux individus de la population, par toutes les voies d'irradiation, dépassera 30 mrem, compte tenu de la durée prévisible d'exposition au rayonnement direct. La durée d'exposition prévisible doit être établie sur la base d'hypothèses prudentes et en tenant compte des particularités locales.

Les limites d'émissions fixées dans l'autorisation se rapportent à l'exploitation normale. Par ailleurs, la limite annuelle englobe les émissions en cas de dérangement.

\subsection{Dose collective de la population en cas d'exploitation normale et en cas de dérangement}

Si les limites d'émissions radioactives sont respectées, la dose collective qui en résultera ne représentera pour la population qu'une modeste fraction

(1) Voir aussi la publication CIPR n०22.

(2) Voir en 5.1. la définition des états de fonctionnement d'une centrale. 
de celle due au rayonnement naturel, de sorte qu'il est superflu, à l'heure actuelle, de fixer une limite quantitative à la dose collective de la population.

\subsection{Dose en cas d'incidents et d'accidents}

Une centrale nucléaire doit être conçue de telle manière que:

- en cas d'incident, il n'y ait pas lieu de prévoir une dose supérieure à 100 mrem par individu de la population avoisinante;

- en cas d'accident, il n'y ait pas lieu de s'attendre, même sur la base d'un calcul pessimiste, à une dose supérieure à 10 rem par individu de la population avoisinante.

\section{PROTECTION DU PERSONNEL D'UNE CENTRALE NUCLEAIRE}

\subsection{Limitation de l'irradiation de personnes exposées dans l'exercice de leur profession en cas de fonctionnement normal et en cas de dérangement}

Conformément aux règles fondamentales énoncées à l'alinéa 2, il convient de démontrer dans la procédure d'autorisation:

a) que les doses maximales admissibles selon OPR, art. 32 à 36 ne seront pas dépassées et,

b) que l'irradiation sera si faible qu'une nouvelle réduction des doses ne se justifierait plus.

\subsection{Doses en cas d'incidents et d'accidents}

La conception d'une centrale nucléaire ainsi que les mesures de précaution prises pour son exploitation doivent viser à assurer au personnel la meilleure protection possible, même en cas d'incident ou d'accident.

\subsection{Dose collective du personnel}

Durant le fonctionnement normal ainsi qu'en cas de dérangement, la dose collective (personnel interne et externe) doit être maintenue au niveau le plus bas qu'il soit raisonnablement possible d'obtenir. Une dose collective supérieure à 400 hommes.rems par an et par réacteur nécessite l'approbation de l'autorité de contrôle.

\section{DEFINITIONS}

\subsection{Etats de fonctionnement d'une centrale nucléaire (')}

(Les probabilités indiquées dans les définitions le sont à titre d'éclaircissement et ne constituent qu'une estimation des ordres de grandeur).

(1) Extrait de la publication de la Commission fédérale pour la sécurité des installations atomiques CSA 22/2 "Definitionen von Fachausdrücken für Kernkraftwerke mit Leichtwasserreaktoren”, éd. 1978. 


\section{Fonctionnement normal}

Le fonctionnement normal englobe tous les états de fonctionnement d'une centrale nucléaire dans le cadre de certaines limites d'exploitation et de prescriptions spécifiques, notamment durant le fonctionnement (à pleine charge ou à charge partielle), durant le démarrage et la mise à l'arrêt, durant l'arrêt, les contrôles et inspections, les opérations d'entretien ou de renouvellement du combustible.

\section{Dérangement}

Etats de fonctionnement s'écartant de la normale et qui peuvent se produire une ou plusieurs fois au cours de l'activité d'une installation (probabilité $>10^{-2}$ par année-réacteur). Etant donné les dispositions prises, ils ne peuvent entraîner aucun dommage à des équipements déterminants pour la sécurité, ni provoquer de doses notables aux environs de la centrale.

\section{Incidents}

Perturbations de faible probabilité $\left(10^{-2}\right.$ à $10^{-4}$ par année-réacteur), causant des dommages tels que, grâce à la conception de la centrale, il n'en résulte aucune atteinte notable à des personnes et à des biens d'autrui en dehors de l'aire de la centrale nucléaire.

\section{Accidents}

Perturbations graves, de très faible probabilité $\left(10^{-4}\right.$ à $10^{-6}$ par annéeréacteur), pouvant endommager l'installation et entraîner la libération d'assez grandes quantités de substances nocives radioactives; la conception de l'installation est, cependant, telle que les effets d'un accident sur des personnes et des biens d'autrui en dehors de l'aire de la centrale restent étroitement limités.

\subsection{Dose}

Sauf autre indication, on entend par dose l'équivalent de dose.

Pour les personnes professionnellement exposées aux radiations, les doses indiquées sont réparties en dose externe par irradiation globale (valeur indiquée par le dosimètre personnel), dose externe par irradiation d'une partie du corps et incorporations.

La dose collective pour des personnes exposées dans l'exercice de leur profession est la somme des doses par irradiation globale.

Pour des individus de la population, la dose est calculée en rems selon la formule:

$$
D=D_{G}+5(\text { rem }) \quad \sum_{i} \frac{D_{i}}{D_{i, \max }}
$$

où
$\mathrm{D}_{\mathrm{G}}$
: dose externe par irradiation globale d'une personne (en rems) prove- nant de nucléides émettant un rayonnement gamma dans le panache d'effluents, ou d'émetteurs gamma déposés au sol.
$\mathrm{D}_{\mathrm{i}} \quad$ : dose de l'organe $\mathrm{i}$, causée par inhalation ou ingestion de nucléides.
$D_{i}$, max: dose maximale tolérée pour l'organe i, selon OPR, art. 34 et art. 44, ch. 2. 